\title{
Osseodensification in Implant Dentistry for Ridge Expansion and Transcrestal Maxillary Sinus Augmentation: Case Report
}

\author{
Ahmed A Elsayyad* \\ Department of Prosthetic Dentistry, Faculty of Oral and Dental Medicine, Cairo University, Egypt \\ *Corresponding author: Ahmed A Elsayyad, Department of Prosthetic Dentistry, Faculty of Oral and Dental Medicine, \\ Cairo University, Egypt
}

\begin{tabular}{l}
\hline ARTICLE INFO \\
\hline Received: 㗀 March 09, 2020 \\
Published: 豐 March 16, 2020 \\
\hline
\end{tabular}

Citation: Ahmed A Elsayyad. Osseodensification in Implant Dentistry for Ridge Expansion and Transcrestal Maxillary Sinus Augmentation: Case Report. Biomed J Sci \& Tech Res 26(4)-2020. BJSTR. MS.ID.004378.

\begin{abstract}
Osseodensification has been a subject of interest in the research arena. It employs densah bur, with negative rake angle and counterclockwise motion, to perform nonsubtractive drilling. In the presence of copious irrigation, it can push the bone trabeculae into the osteotomy instead of leaving it. This compaction can increase bone density, bone volume and subsequently enhance implant stability. The aim of this study is to report a case using densah bur successfully for osseous densification and crestal maxillary sinus augmentation. Further clinical trials are required to validate these findings and confirm whether it can be used for ridge expansion.
\end{abstract}

Keywords: Osseodensification; Densah Bur; Counterclockwise Drilling; Ridge Expansion, Maxillary Sinus Lift

\section{Introduction}

Sufficient amount of pristine bone surrounding dental implants is perquisite for successful osseointegration. Unfortunately, maxillary sinus pneumatization and posterior alveolar ridge atrophy occur rapidly following extraction of maxillary posterior teeth. Attempts have been made to increase primary mechanical and secondary biologic stability via improving bone quality and quantity. Bone expanders have been used to increase bone width, but at the expense of causing trabecular microfractures with subsequent bone resorption [1]. Techniques to improve bone height in the posterior maxilla have been introduced including maxillary sinus lift whether direct (lateral window) or indirect (crestal) approach. The direct approach is invasive with greater possibility of patient morbidity in contrast to the crestal approach. However, the latter technique poses a $24 \%$ risk of sinus membrane perforation due to impaired visibility [2].

Another technique, adopting the concept of osseodensification, was introduced in 2014 and seems to be promising solution to the limitations of the techniques of enhancing bone quantity and quality.
It is based on a special bur with counterclockwise drilling, called Densah bur (Versah, USA). Non-subtractive reverse drilling, using copious amounts of irrigation, can push the cut bony trabeculae into the osteotomy instead of leaving it. This compression increases bone width two to three folds and increases bone density compared to regular drilling [3]. Elsayyad and Osman concluded that despite being very promising, the studies favoring osseodensification are mainly animal studies with high risk of bias [4]. This calls for the need for conducting human studies of higher evidence. Therefore, the aim of this study is to report a case employing osseodensification to enhance bone quality and quantity in atrophic posterior maxillary ridge. Ridge expansion along with crestal sinus lift using densah bur was accompanied with simultaneous implant placement.

\section{Case Representation}

A 40-year-old female patient was referred to the Prosthodontics department in our School of Dentistry in January 2018. The patient's informed consent was obtained to include her photographs and radiographs in our study. Intra-oral examination of the maxillary 
arch (Figure 1) revealed multiple carious lesions which were restored with composite resin. Remaining roots of maxillary left premolars and first molar as well as non-restorable maxillary left second molar, due to extensive subgingival caries, were extracted. Implants insertion was delayed for 6 months after extraction to allow for complete remodeling of the extraction sockets. CBCT requested two weeks prior to implants' placement showed slightly deficient bone bucco-lingually in the maxillary left first premolar region (Figure 2A). Furthermore, diminished bone height (4.19 $\mathrm{mm}$ ) was evident in the left second molar region (Figure 2B). Hence, densah bur was used for ridge expansion and crestal sinus lift in the left first premolar and second molar respectively.

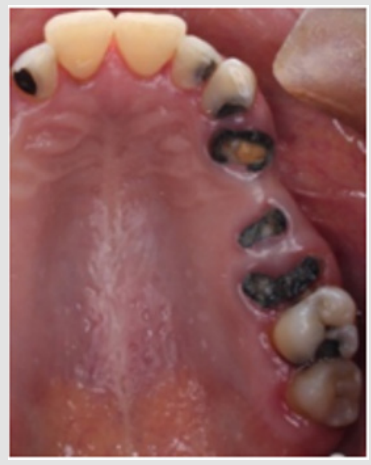

Figure 1: Intra-oral maxillary occlusal view showing carious right and left lateral incisors as well as left canine, remaining roots of left premolars and first molar and nonrestorable left second molar.
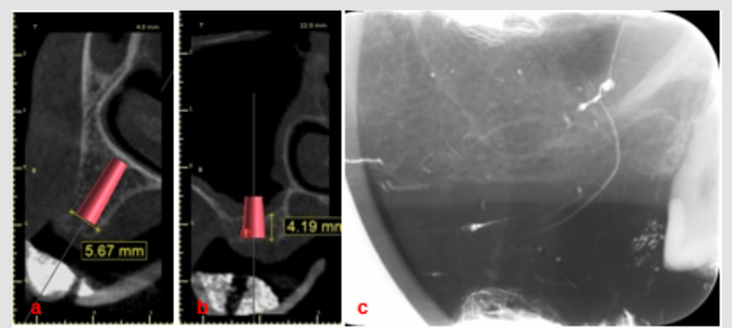

Figure 2: Radiographs requested 6 months post-extraction. a. CBCT showing slightly deficient bone width for implant in the first premolar region,

b. $\mathrm{CBCT}$ showing maxillary sinus pneumatization and reduced bone height for implant in the second molar region,

c. Peri-apical radiograph showing severe sinus pneumatization.

Peri-apical $\mathrm{x}$-rays were taken prior to implant surgery for comparative purposes (Figure 2C). In the first premolar region, drilling using Densah burs (VS8) proceeded under counterclockwise pumping motion (Figure 3A). During insertion of the final drill $(3.5 \times 11.5 \mathrm{~mm})$, fenestration of the buccal wall of the osteotomy occurred. Implant $(4 \times 11.5 \mathrm{~mm})$ (Neobiotech, Korea) was inserted and the dehiscence was managed by grafting with DM Bone (Neobiotech, Korea) and T-Gen membrane (SK bioland Co., Korea) (Figure 3B). In the second molar region, pilot drill was inserted $1 \mathrm{~mm}$ short of the sinus membrane. Particulate DM Bone was packed into the prepared osteotomy. Sequential drilling then proceeded employing $200 \mathrm{rpm}$ speed without irrigation, so that the densah bur can push the grafts coronally (Figure 4A \& 4B). The compacted grafts lifted the sinus membrane gradually until reaching the desired height $(8 \mathrm{~mm})$, followed by implant $(4 \times 7.3$ $\mathrm{mm}$ ) insertion (Figure 4C). Implant stability quotient (ISQ) of both implants was measured on the day of surgery, 3 and 6 months postoperatively (Ostell, Sweden) (Table 1). After 6 months, the implants were restored with porcelain fused to metal screw-retained bridge using CCM UCLA abutments (Neobiotech, Korea) (Figure 5A \& 5B). The patient was followed-up for a period of six months with no complications.
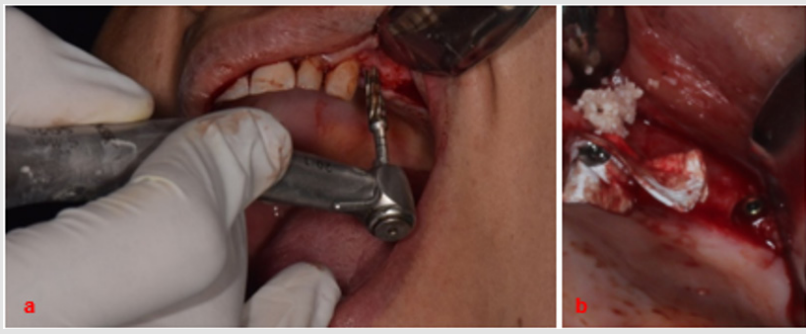

Figure 3: Implant bed preparation in the first premolar region.

a. Densah bur counter-clockwise drilling for ridge expansion,

b. Buccal wall fenestration occurred which was managed using particulate bone graft and membrane.
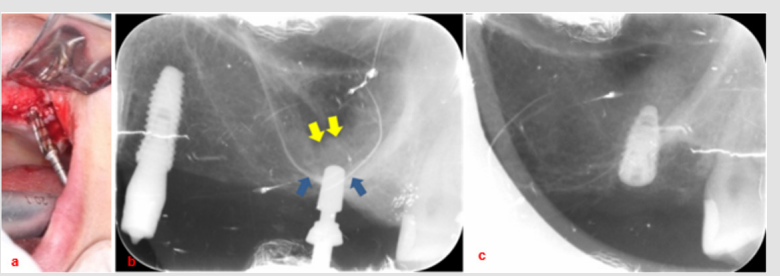

Figure 4: Implant bed preparation in the second molar region along with crestal sinus lift.

a. Densah bur compressing the bone graft into the osteotomy for crestal sinus lift,

b. Sequential drilling produced densification of the sinus floor (blue arrow) with the compressed bone graft (yellow arrow) elevating the sinus membrane,

c. Implant $(4 \times 7.3 \mathrm{~mm})$ inserted into prepared osteotomy. 


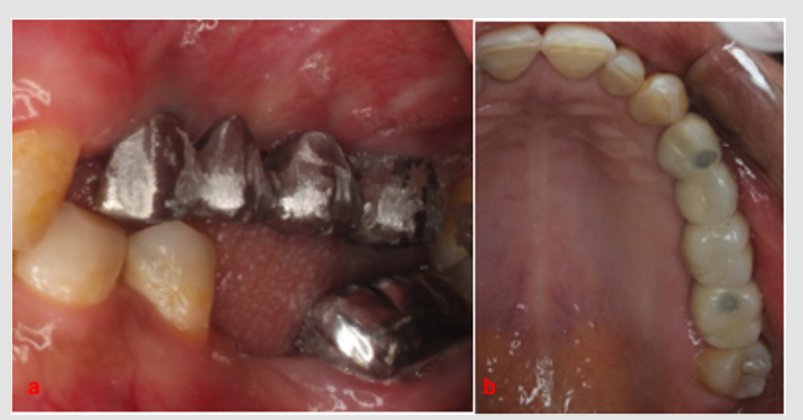

Figure 5: Screw-retained porcelain fused to metal bridge.

a. A: Intra-oral profile view of the metal try-in,

b. B: Occlusal view showing final bridge with composite sealing the access holes.

Table 1: ISQ values measured at 0,3 and 6 months.

\begin{tabular}{|c|c|c|c|c|c|c|c|c|c|c|c|c|}
\hline $\begin{array}{c}\text { Time } \\
\text { (months) }\end{array}$ & \multicolumn{4}{|c|}{0} & \multicolumn{4}{|c|}{3} & \multicolumn{4}{|c|}{6} \\
\hline \multirow{2}{*}{$\begin{array}{c}\text { First } \\
\text { premolar }\end{array}$} & B & $\mathbf{P}$ & $\mathbf{M}$ & D & B & $\mathbf{P}$ & $\mathbf{M}$ & D & B & $\mathbf{P}$ & $\mathbf{M}$ & D \\
\hline & 17 & \multicolumn{3}{|c|}{70} & \multicolumn{4}{|c|}{78} & & & \multicolumn{2}{|c|}{79} \\
\hline \multirow{2}{*}{$\begin{array}{l}\text { Second } \\
\text { molar }\end{array}$} & B & $\mathbf{P}$ & $\mathbf{M}$ & D & B & $\mathbf{P}$ & $\mathbf{M}$ & D & B & $\mathbf{P}$ & $\mathbf{M}$ & D \\
\hline & 25 & 28 & 30 & 32 & \multicolumn{4}{|c|}{43} & 69 & \multicolumn{2}{|c|}{72} & 71 \\
\hline
\end{tabular}

Abbreviations: B: Buccal, P: Palatal, M: Mesial, D: Distal

\section{Discussion}

The aim of this study is to report a case employing densah bur for the dual purpose of osseodensification and increasing bone quantity. With the aid of counterclockwise drilling, it can compresses the autografts against the sides and the apex of the implant bed [3]. Resonance frequency analysis, expressed as ISQ values, can be used to evaluate initial stability. ISQ $>70$ indicates high stability allowing for safe immediate loading [5]. Based on the preliminary results obtained from our study, densah bur can be a valuable tool for osseodensification. This was evident by the high ISQ values (70) measured on all surfaces of maxillary first premolar except for the buccal surface where fenestration occurred. However, this fenestration didn't negatively affect ISQ values measured 3 and 6 months postoperatively, with peak values of 78 and 80 respectively. Similar positive results was confirmed on animal models [6]. Despite very low ISQ values in the second molar region which may be expected due to diminished bone height $(4 \mathrm{~mm})$, they increased dramatically at 6 months with peak values of 72 . Counter-clockwise motion at low speed without irrigation enabled compressed bone graft to lift the sinus membrane. This approach can be more favorable to patients when compared to traumatic hammer and mallet use [2]. In our study, about $4 \mathrm{~mm}$ of the sinus membrane was elevated which suggests that densah bur can be a predictable alternative to osteotomes. This finding is in agreement with the study conducted by Huwais et al., reporting 97\% implant survival over 5-year follow-up period after using densah bur for transcrestal sinus augmentation [7]. Despite following the "Plus1"
Protocol recommended by the manufacturer for ridge expansion, buccal wall fenestration occurred [8]. This calls for conducting clinical trials to investigate the preliminary finding of this case report, regarding the questionable effectiveness of densah bur in ridge expansion. This case report gives an overall view about the possibility of using densah bur for densifying bone, ridge expansion and crestal maxillary sinus lift. It is limited by its short follow-up period, lack of postopertative radiographs because the patient became pregnant in her first trimester. Clinical trials are required to validate the preliminary findings of this study.

\section{Conclusion}

Within the limitations of this study, densah bur can be a promising tool for crestal sinus lift, even when diminished bone height is available, and osseous densification. Whether it can reliably replace bone expanders for ridge expansion is a question that still merits further investigations. Well-designed human clinical trials of long follow-up periods are required to validate or refute these findings.

\section{Ethical Statement}

1. Funding: (if any): No sources of funding were available to conduct this study.

2. Conflict of Interest: The authors declare that there is no conflict of interest.

3. Ethical approval: Approval by the Ethics committee was obtained for the conduct of this study. 
4. Informed consent: Patient's informed consent was obtained to include her photographs and radiographs in our study.

\section{Authors' contributions}

Both authors (A.E, N.A):

1. Have made substantial contributions to conception and design of the study.

2. Have drafted the article and revised it critically.

3. Have approved the final version to be published.

\section{References}

1. Büchter A, Kleinheinz J, Wiesmann HP, Kersken J, Nienkemper M, et al. (2004) Biological and biomechanical evaluation of bone remodelling and implant stability after using an osteotome technique. Clin Oral Implants Res 16(1): 1-8.

2. Toffler M, Rosen PS (2015) Complications with transcrestal sinus floor elevation: Etiology, prevention, and treatment. Dental Implant Complications: Etiology, Prevention, and Treatment pp. 427-456.

3. Huwais S, Meyer EG (2017) A Novel Osseous Densification Approach in Implant Osteotomy Preparation to Increase Biomechanical Primary
Stability, Bone Mineral Density, and Bone-to-Implant Contact. Int J Oral Maxillofac Implants 32(1): 27-36.

4. Elsayyad AA, Osman RB (2019) Osseodensification in Implant Dentistry: A Critical Review of the Literature. Implant Dent 28(3): 306-312.

5. Bornstein MM, Hart CN, Halbritter SA, Morton D, Buser D (2009) Early Loading of Nonsubmerged Titanium Implants with a Chemically Modified Sand-Blasted and Acid-Etched Surface: 6-Month Results of a Prospective Case Series Study in the Posterior Mandible Focusing on Peri-Implant Crestal Bone Changes and Implant Stability Quotient (ISQ) Values. Clin Implant Dent Relat Res 11(4): 338-347.

6. Lahens B, Neiva R, Tovar N, Alifarag AM, Jimbo R, et al. (2016) Biomechanical and histologic basis of osseodensification drilling for endosteal implant placement in low density bone. An experimental study in sheep. J Mech Behav Biomed Mater 63: 56-65.

7. Huwais S, Mazor Z, Ioannou AL, Gluckman H, Neiva R (2018) A Multicenter Retrospective Clinical Study with Up-to-5-Year Followup Utilizing a Method that Enhances Bone Density and Allows for Transcrestal Sinus Augmentation Through Compaction Grafting. Int J Oral Maxillofac Implants 33(6): 1305-1311.

8. Densah ${ }^{\circledR}$ Bur Surgical Technique Manual.
ISSN: 2574-1241

DOI: $10.26717 / B J S T R .2020 .26 .004378$

Ahmed A Elsayyad. Biomed J Sci \& Tech Res

(c) (i) This work is licensed under Creative

Submission Link: https://biomedres.us/submit-manuscript.php Commons Attribution 4.0 License

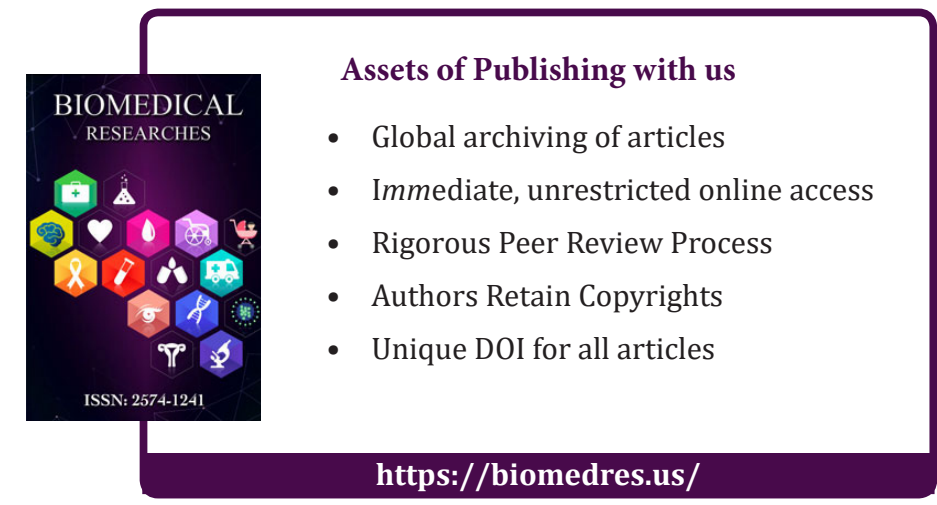

